\title{
Research on intelligence analysis system of subway counterterrorism video surveillance
}

\author{
Shuqi Li \\ School of Humanities and Economic Management, China University of Geosciences, Beijing \\ 100083, China. \\ huner2011@foxmail.com
}

Keywords: Subway, Anti-terrorism, Intelligence, Algorism

\begin{abstract}
With the development of the capital's subway system and rampant terrorist activities at home and abroad, the subway has become one of the important targets of violence terrorist attacks. Although Beijing subway system has the initially constructed the video surveillance systems currently, there are deficiencies in intelligence analysis. This study pans to use the algorithm innovation to integrate intelligent analysis technology in the video surveillance system, which can enhance the overall level of anti-terrorism and counter-terrorism in the subway.
\end{abstract}

\section{Introduction}

With the rapid development in several years, the Beijing subway has become one of the world's busiest metro systems. Once the subway process runs accident, it will cause huge casualties and property losses. Then, terrorists turn target to the subway system with huge flow of people. While our society is stable in the whole, economic develops healthily, the terrorist organizations and terrorists continue to create disturbances and terrorist attacks all over the country. As the capital city, Beijing itself is the focus of the target area of terrorist attacks, how to prevent terrorist activities in subway system has become an important issue which is in urgent need of research [1-2]. With the crowd, narrow space, lighting conditions and other complex influences in the subway environment, the existing imaging systems cannot fully make effects. The study of subway counterterrorism video surveillance intelligence analysis system mainly aims to the existing subway supervisory system in terms of the presence of anti-terrorism and counter-terrorism technical problems [3-5], such as low levels of artificial intelligence, cannot immediately suspicious circumstances. In complex environments such as many people, it is difficult for instant face image positioning, extraction, analysis and identification. The lighting environment in subway system is complex, there are many reflective, shadows, highlights and other interference, reducing the recognition accuracy, cannot make early warning of abnormal situation occurring in real time, and monitoring some of its other technical problems and the like. Video monitoring intelligent analysis system [6-8] is able to better help find hidden risk points and suspicious people, and make anticipation warning about intelligence information collected, thereby to provide intelligence support to prevent the occurrence of terrorist attacks.

\section{The main function of system}

Technical difficulties of intelligent video surveillance analysis are in target detection and target tracking algorithm, target detection using background subtraction method. First, use live video for the single Gaussian background modeling and subtraction with the current frame and the background frame. The target area with large differences can be considered to be within the scene movement. It generally provides relatively more comprehensive characterization data of moving target, but is also particularly sensitive to the dynamics of the scene. To overcome the interference in the shadows of the background of this algorithm, use color vector and texture features to eliminate shadows. While taking into account the importance of pedestrian detection, tracking and behavior analysis, systematic study adds a special pedestrian detection on the basis of foreground extraction, and in complex scenes, pedestrian detection rate and false alarm rate is difficult for 
effective control at the same time, and complex methods such as HOG + latent SVM, the use of local deformation template collection separable support vector machine to infer implicit global location of people although makes better detection results, for a huge amount of computation, it is difficult to get real-time applications. Use the integral channel features to provide a lot of candidate features, combined with AdaBoost algorithm to select the sub-feature which has the ability to identify, and ultimately achieve a fast and accurate detection of human.

Target tracking in system uses a particle filter framework algorithm. The system includes software design and embedded algorithms, and systems research is divided into front-end and back-end equipment to detect intelligent platform for smart handling which consist of sub-total of eight items, including the integration of rule algorithms design and systems.

1). Personnel illegal entry in key areas

2). Focus items are illegally removed

3). Personnel long stay in key areas

4). Facial recognition of dangerous staff

5). The abnormal crowd gather in public places

6). Confusion caused by fights and other acts in public places

7). Unidentified items are discarded in public places

8). Face detection

\section{Architecture diagram of video surveillance intelligence analysis system}

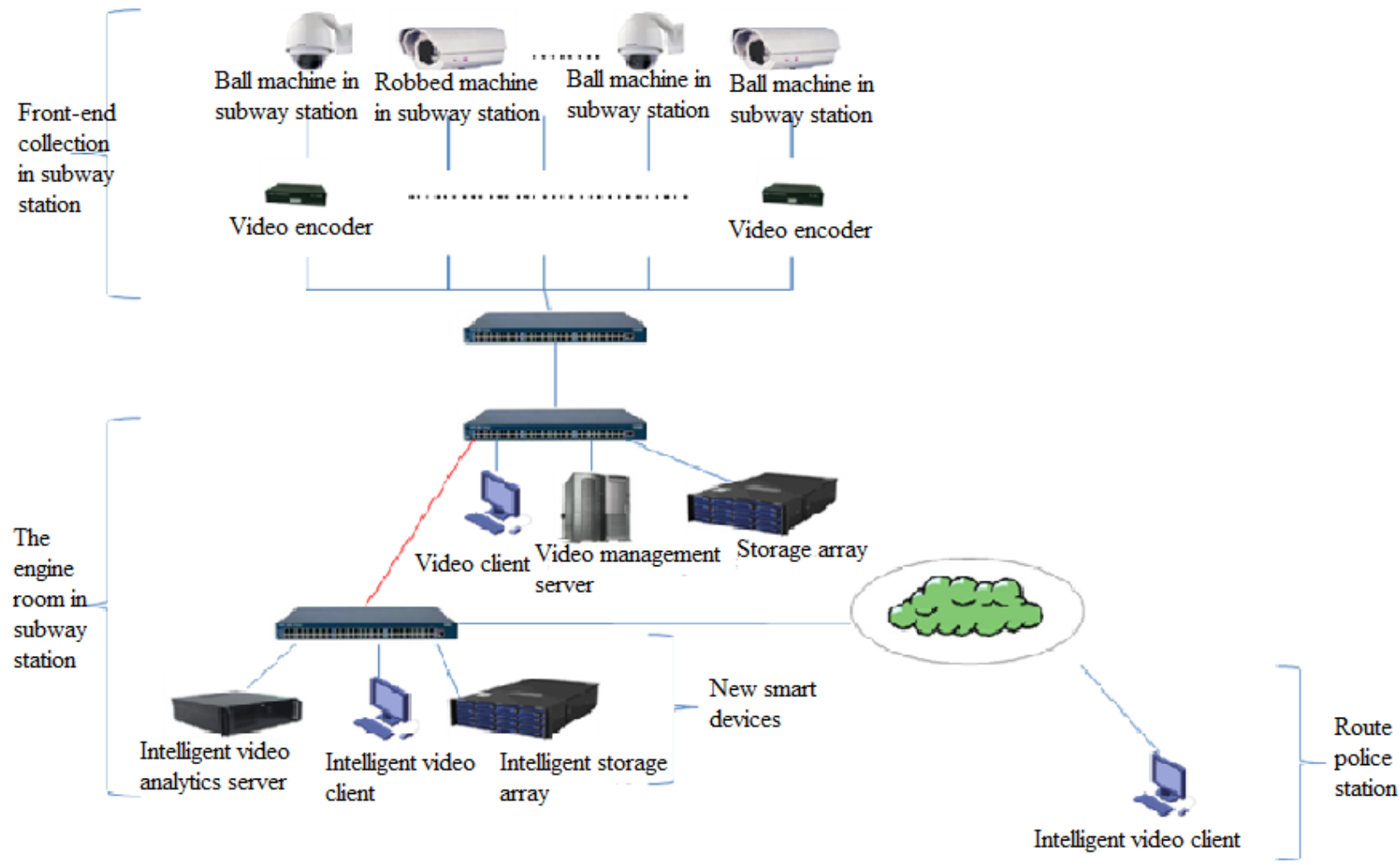

Fig. 1 Architecture diagram of video surveillance intelligence analysis system

As shown in Fig. 1, before the system front-end network, video devices with embedded analysis algorithm is a powerful DSP chip processing while performing codec processing, use DSP processing resources to achieve surplus intelligent video analysis. DSP analysis capability is composed of a variety of algorithms, different algorithms used in different scenarios being, such as facial recognition algorithm, intrusion recognition algorithm, target tracking and super-resolution image enhancement algorithms, and the needs of various application scenarios with the changes in the specific environment will change. Use video information directly for intelligent analysis to achieve the appropriate intelligent video capabilities. 


\section{Algorithm and implementation of intelligent video surveillance analysis system}

Personnel illegal entry in key areas. Key areas in achieving the target detection use AdaBoost with integral channel features to achieve pedestrian detection, on this basis, under the framework of particle filter combined with online learning and testing of pedestrian tracking algorithm. Here introduce intrusion detection decision rules.

Let the set area is $\mathrm{R}$, the moving target is M. Relationship between the two is mainly the following three, as shown in Fig. 2.
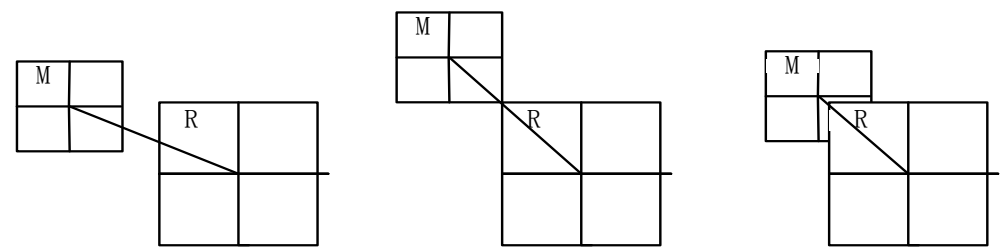

Fig. 2 Relative location of target and set area

According to the target detecting to get the coordinates of moving targets (topy, bottomy, leftx, rightx) and center coordinates (x,y), and the width and height. Suppose now we have obtained border coordinates outside the set area, then according to equation (1), we can calculate center of gravity coordinates $(\mathrm{X}, \mathrm{Y})$, the width $\mathrm{W}$ and the height $\mathrm{H}$.

$$
\left.\begin{array}{l}
x=\frac{\text { left } x+\text { right } x}{2} \\
y=\frac{\text { topy }+ \text { bottomy }}{2} \\
\text { width }=\text { right } x-\text { left } x \\
\text { height }=\text { topy }- \text { bottomy }
\end{array}\right\}
$$

Based on the above analysis, we can get intrusion detection rules:

$$
\left\{\begin{array}{cc}
|X-x|<\frac{W+\text { width }}{2} \text { and }|Y-y|<\frac{H+\text { height }}{2} & \text { Yes } \\
\text { else } & \text { No }
\end{array}\right.
$$

Focus items are illegally removed.

From Fig. 3, derelict and goods illegally removed detection is divided into three modules: Background generation module, analysis module and trigger module. 


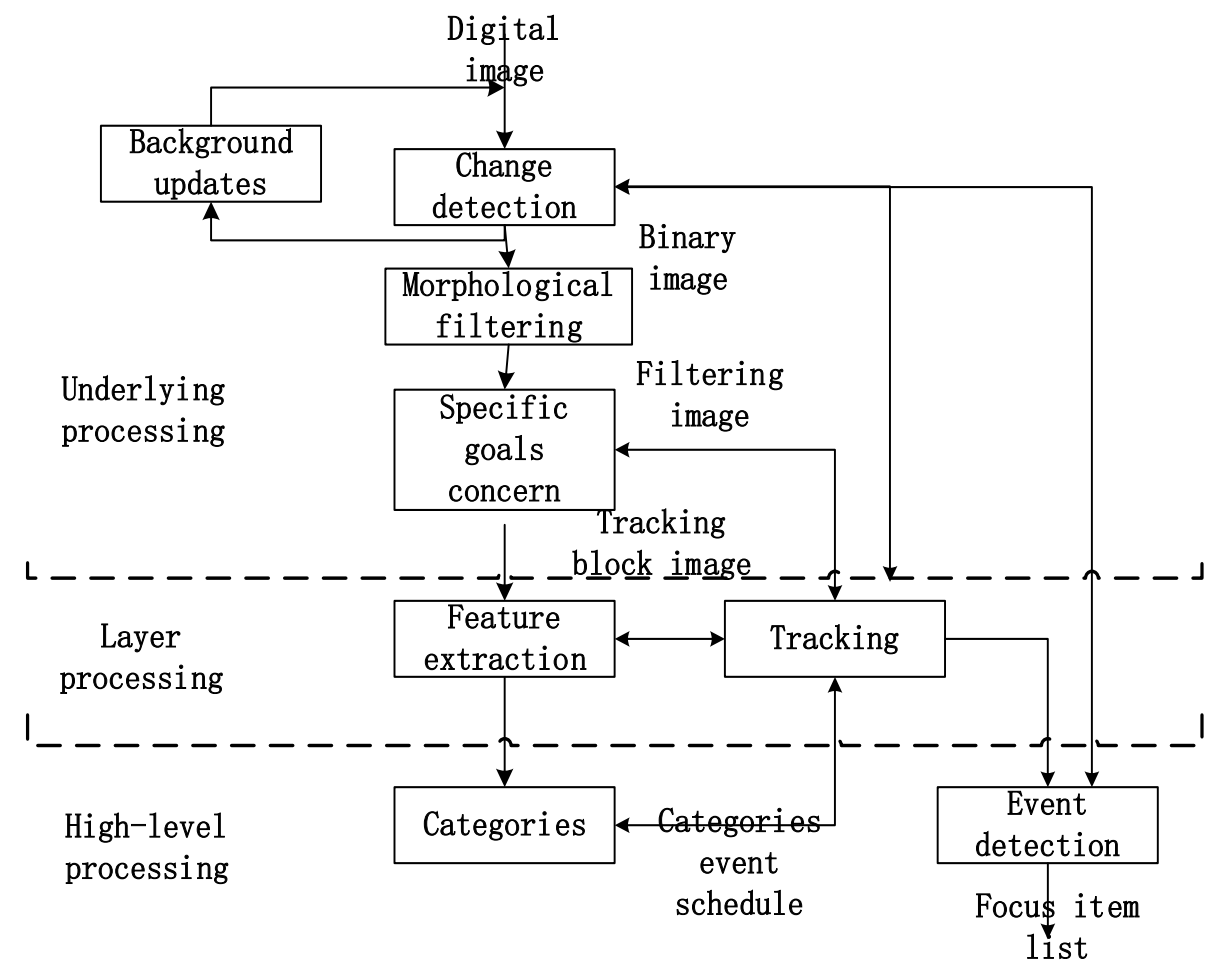

Fig. 3 Derelict and goods illegally removed detection

Personnel long stay in key areas. In the implementation, using integral channel features combined with AdaBoost to achieve pedestrian detection, combined with particle filter framework under the call on the basis of online learning and testing of pedestrian tracking algorithm (see Fig. 4). Then analyze to determine the locus of decision rules based on prolonged stay staffed events.

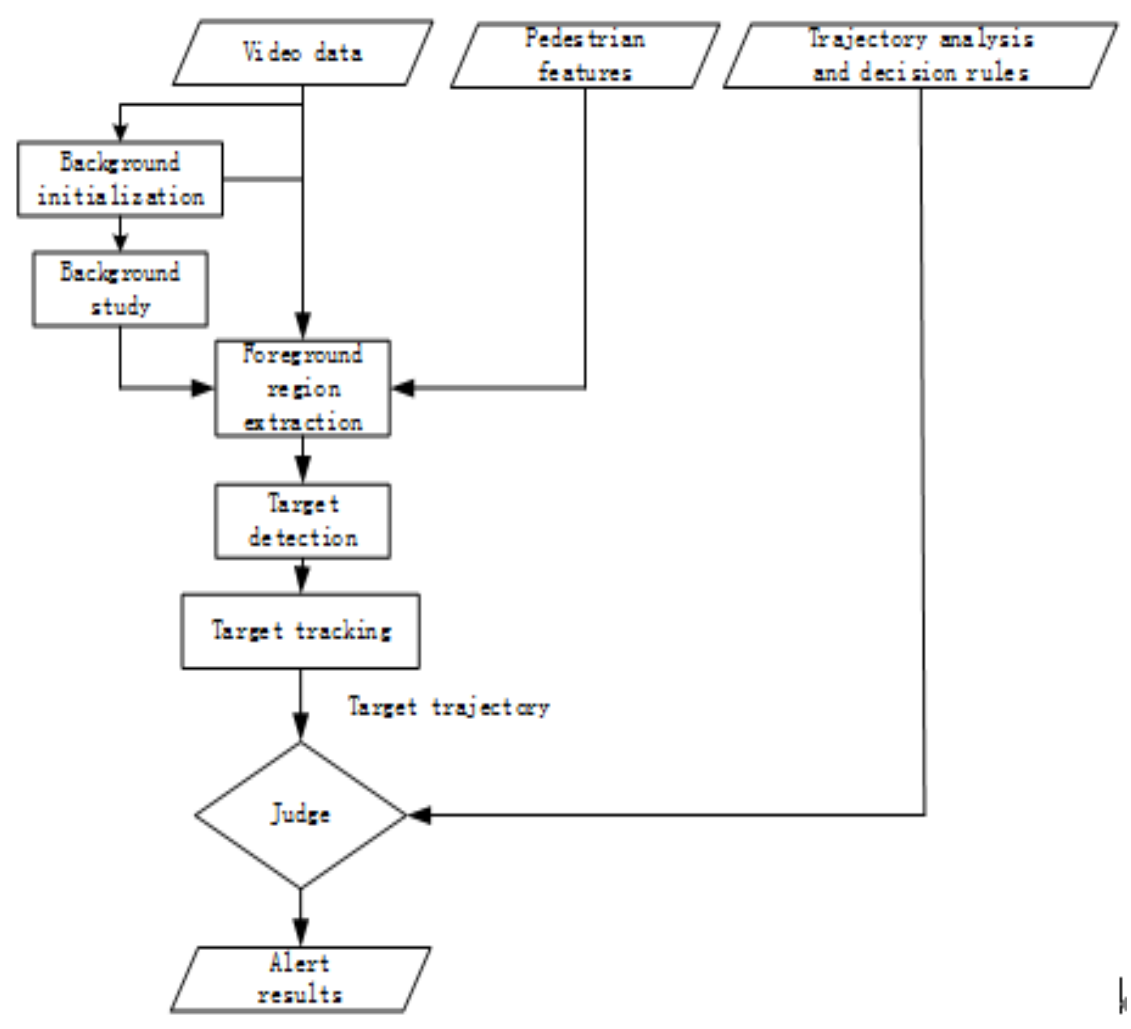

Fig. 4 Learning and testing of pedestrian tracking algorithm 


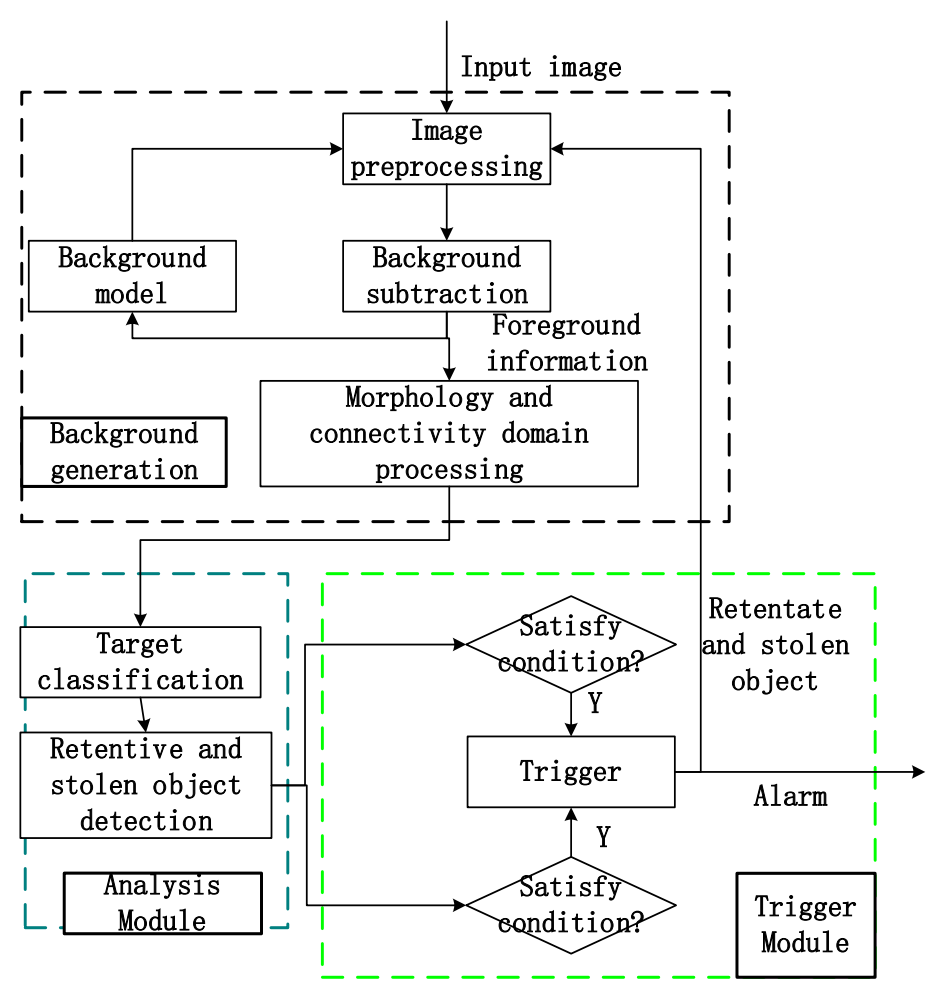

Fig. 5 Identifying unknown items in public places

Unknown items are discarded in public places. There are 3 kinds of scenes. a) no object or only moving objects; b) only retentive objects; c) both moving objects and retentive objects. We do not need test the properties of target objects and identify the next scenes directly if the scenes belong to a). When scenes of b) occur, we can test and segment the target objects based on background subtraction. And we can remove the moving objects if scenes of c) happen. The process of identifying unknown items in public place is shown in Fig. 5.

\section{Conclusion}

Adopt intelligent analysis equipment, timely collect feedback suspected security incidents confusion, to attain the new mode of "routine patrol" combined with anti-terrorism technology, it will be greatly beneficial to carry out the daily activities of the subway security, significantly improve the combat effectiveness of anti-terrorism and counter-terrorism.

Relying on the expanded research to gradually apply intelligent video analysis system judgment to build monitoring and field personnel on patrol, the linkage mechanism of armed personnel around and guard personnel, maintaining rail traffic order, the protection of public safety travel, effectively preventing all kinds of unexpected events to provide technical support for the construction of "Green Beijing”.

\section{References}

[1] Loukaitou-Sideris A, Taylor B D, Fink C N Y. Rail Transit Security in an International Context Lessons from Four Cities. Urban Affairs Review, 2006, 41(6): 727-748.

[2] Miguel-Tobal J.J., Vindel A C, Iruarrízaga I, et al. Psychopathological repercussions of the March 11 terrorist attacks in Madrid. Clínica y Salud, 2004, 15(3): 293-304.

[3] Paragios N, Ramesh V. A MRF-based approach for real-time subway monitoring//Computer Vision and Pattern Recognition, 2001. CVPR 2001. Proceedings of the 2001 IEEE Computer Society Conference on. IEEE, 2001, 1: I-1034-I-1040 vol. 1. 
[4] Wang J., Wang C., Chen Z., et al. Subway Integrated Monitoring System Under the Framework of ITS//International Conference on Transportation Engineering 2009. ASCE, 2009: 2079-2083.

[5] Liu X., Wei S.Y., Gao Q., et al. Subway Monitoring System Based on TM30 Georobot Research and Implementation. Advanced Materials Research, 2012, 368: 2109-2116.

[6] Lipton A, Kanade T, Fujiyoshi H, et al. A system for video surveillance and monitoring. Pittsburg: Carnegie Mellon University, the Robotics Institute, 2000.

[7] Lee L, Romano R, Stein G. Introduction to the special section on video surveillance[J]. IEEE Transactions on Pattern Analysis and Machine Intelligence, 2000, 22(8): 745.

[8] Tian Y L, Lu M, Hampapur A. Robust and efficient foreground analysis for real-time video surveillance//Computer Vision and Pattern Recognition, 2005. CVPR 2005. IEEE Computer Society Conference on. IEEE, 2005, 1: 1182-1187. 